Bull. Korean Math. Soc. 44 (2007), No. 1, pp. 61-71

\title{
POLYGONAL PRODUCTS OF RESIDUALLY FINITE GROUPS
}

\author{
Kok Bin Wong and Peng Choon Wong
}

\begin{abstract}
A group $G$ is called cyclic subgroup separable for the cyclic subgroup $H$ if for each $x \in G \backslash H$, there exists a normal subgroup $N$ of finite index in $G$ such that $x \notin H N$. Clearly a cyclic subgroup separable group is residually finite. In this note we show that certain polygonal products of cyclic subgroup separable groups amalgamating normal subgroups are again cyclic subgroup separable. We then apply our results to polygonal products of polycyclic-by-finite groups and free-byfinite groups.
\end{abstract}

\section{Introduction}

The polygonal products of groups were introduced by Karrass, Pietrowski and Solitar [6] in their study of the subgroup structure of the Picard group $\operatorname{PSL}(2, \mathrm{Z}[\mathrm{i}])$. By using their results, Brunner, Frame, Lee and Wielenberg [3] characterized all the torson-free subgroups of finite index in the Picard group. Polygonal products also form a large subclass in the class of one-relator products of cyclic groups. For certain groups in the above class, Fine, Howie and Rosenberger [4] had proved a Freiheitssatz but the word problem and residual finiteness are still unknown.

Unlike the generalized free products of groups, the residual finiteness of polygonal products are little known even when the amalgamated subgroups are cyclic. In addition, polygonal products do not have many residual properties. In [1] Allenby and Tang showed that the polygonal products of finitely generated free abelian groups amalgamating cyclic subgroups with trivial intersections are residually finite. But in the same paper, Allenby and Tang also gave an example of a polygonal product of finitely generated nilpotent groups of class 2 amalgamating cyclic subgroups which is not residually finite.

In [2], G. Baumslag proved that the generalized free products of two polycyclic-by-finite groups amalgamating a central subgroup are residually finite.

Received September 29, 2005.

2000 Mathematics Subject Classification. Primary 20E06, 20E26, 20F18; Secondary $20 \mathrm{~F} 05$.

Key words and phrases. subgroup separable, polygonal products, polycyclic-by-finite groups, free-by-finite groups, abelian groups.

(C)2007 The Korean Mathematical Society 
More recently, Kim [7] has shown that the polygonal products of polycyclicby-finite groups amalgamating central subgroups are cyclic subgroup separable (or $\pi_{c}$ for short) and hence residually finite. In this note we will prove that the polygonal products of polycyclic-by-finite groups amalgamating normal subgroups are $\pi_{c}$ and hence residually finite. More precisely, we shall show that the polygonal products of subgroup separable groups amalgamating finitely generated normal subgroups are $\pi_{c}$. Thus the polygonal products of polycyclicby-finite groups and free-by-finite groups amalgamating normal subgroups are $\pi_{c}$.

The notations used here are standard. In addition the following notations will be used for any group $G$ :

$N \triangleleft_{f} G$ means $N$ is a normal subgroup of finite index in $G$.

$\|x\|$ means the usual generalized free product length of $x$.

$A_{H}^{*} B$ denotes the generalized free product of $A$ and $B$ amalgamating a subgroup $H$.

\section{Preliminaries}

We begin with the following definition and theorems.

Definition 2.1. A group $G$ is called $H$-separable for the subgroup $H$ if for each $x \in G \backslash H$, there exists $N \triangleleft_{f} G$ such that $x \notin H N$.

$G$ is called $H K$-separable for the subgroups $H, K$ if for each $x \in G \backslash H K$, there exists $N \triangleleft_{f} G$ such that $x \notin H K N$.

$G$ is termed subgroup separable if $G$ is $H$-separable for every finitely generated subgroup $H$.

$G$ is termed cyclic subgroup separable (or $\pi_{\mathrm{c}}$ for short) if $G$ is $H$-separable for every cyclic subgroup $H$.

It is well known that polycyclic groups and free groups are subgroup separable (Mal'cev [8], M. Hall [5]). Since a finite extension of a subgroup separable group is again subgroup separable, polycyclic-by-finite groups and free-by-finite groups are subgroup separable.

The following theorems will be used in the proof of several theorems.

Theorem 2.2. (Baumslag [2]) Let $G=A_{H}^{*} B$ where $A$ and $B$ are finite. Then $G$ is subgroup separable and hence $\pi_{c}$.

Theorem 2.3. (Kim [7]) Let $G=A_{H}^{*} B$. Suppose that

(a) $A$ and $B$ are $\pi_{c}$ and $H$-separable,

(b) for each $N \triangleleft_{f} H$, there exist $N_{A} \triangleleft_{f} A$ and $N_{B} \triangleleft_{f} B$ such that $N_{A} \cap H=$ $N_{B} \cap H \subset N$.

Then $G$ is $\pi_{c}$. 


\section{Free products amalgamating normal subgroups}

In this section we shall show that the generalized free products of finitely many subgroup separable groups amalgamating normal subgroups with trivial intersections are $\pi_{c}$. We begin with the generalized free products of two groups.

The followings two lemmas (Lemma 3.1, Lemma 3.2) are well known to researchers in this area. However we provide the proof for expository purposes.

Lemma 3.1. Let $H$ be a finitely generated group and $S \triangleleft_{f} H$. Then there exists $f_{H}(S) \subseteq S$ such that $f_{H}(S)$ is a characteristic subgroup of finite index in $H$.

Proof. We define $f_{H}(S)$ as follow: If $S$ is a characteristic subgroup of $H$ then $f_{H}(S)=S$. Suppose $S$ is not a characteristic subgroup of $H$. Let $[H: S]=m$ where $m$ is a positive integer. Since $H$ is finitely generated, the number of subgroups of index $m$ in $H$ is finite. Let $N$ be the intersection of all these subgroups. Then $N$ is a characteristic subgroup of finite index in $H$ and $N \subseteq S$. We then define $f_{H}(S)=N$.

Lemma 3.2. Let $A$ be a subgroup separable group and $H$ be a finitely generated normal subgroup of $A$. If $S \triangleleft_{f} H$ and $S$ is normal in $A$, then there exists $N \triangleleft_{f} A$ such that $N \cap H=S$.

Proof. Since $H$ is finitely generated and $S \triangleleft_{f} H$, then $S$ is also finitely generated. Since $A$ is subgroup separable, then $\bar{A}=A / S$ is residually finite. Now $\bar{H}=$ $H / S$ is finite. Therefore there exists $\bar{N} \triangleleft_{f} \bar{A}$ such that $\bar{N} \cap \bar{H}=\{1\}$. Let $N$ be the preimage of $\bar{N}$. Then $N \cap H=S$.

Lemma 3.3. Let $A$ be a subgroup separable group and $H$ be a finitely generated normal subgroup of $A$. Then for each $S \triangleleft_{f} H$, there exists $f_{H}(S) \subseteq S$ such that $f_{H}(S)$ is a characteristic subgroup of finite index in $H$ and there exists $N \triangleleft_{f} A$ such that $N \cap H=f_{H}(S)$.

Proof. Follows from Lemmas 3.1 and 3.2.

Theorem 3.4. Let $G=A_{H}^{*} B$ where $A$ and $B$ are subgroup separable and $H$ is a finitely generated normal subgroup of $A$ and $B$. Then $G$ is $\pi_{c}$.

Proof. We shall use Theorem 2.3. Since $A, B$ are $\pi_{c}$ and $H$-separable, it is sufficient to show that given any $N_{H} \triangleleft_{f} H$, there exist $N_{A} \triangleleft_{f} A$ and $N_{B} \triangleleft_{f} B$ such that $N_{A} \cap H=N_{B} \cap H \subseteq N_{H}$. Now given any $N_{H} \triangleleft_{f} H$, then by Lemma 3.3, there exists a characteristic subgroup $f_{H}\left(N_{H}\right)$ of $H$ such that $f_{H}\left(N_{H}\right) \subseteq N_{H}$ and there exist $N_{A} \triangleleft_{f} A$ and $N_{B} \triangleleft_{f} B$ such that $N_{A} \cap H=f_{H}\left(N_{H}\right)=N_{B} \cap H$. The result follows from Theorem 2.3.

Next we extend Theorem 3.4 to generalized free products of more than two groups.

Lemma 3.5. Let $A$ be a subgroup separable group and $H_{1}, H_{2}$ be finitely generated normal subgroups of $A$ and $H_{1} \cap H_{2}=\{1\}$. If $S_{1} \triangleleft_{f} H_{1}, S_{2} \triangleleft_{f} H_{2}$ and $S_{1}, S_{2}$ 
are normal in $A$, then there exists $N \triangleleft_{f} A$ such that $N \cap H_{1}=S_{1}, N \cap H_{2}=S_{2}$ and $\mathrm{NH}_{1} \cap \mathrm{NH}_{2}=\mathrm{N}$.

Proof. Since $H_{1}, H_{2}$ are finitely generated and $S_{1} \triangleleft_{f} H_{1}, S_{2} \triangleleft_{f} H_{2}$, then $S_{1}$ and $S_{2}$ are also finitely generated. Therefore $S_{1} S_{2}$ is finitely generated and hence $\bar{A}=A / S_{1} S_{2}$ is residually finite. Since $\overline{H_{1} H_{2}}=H_{1} H_{2} / S_{1} S_{2}$ is finite, there exists $\bar{N} \triangleleft_{f} \bar{A}$ such that $\bar{N} \cap \overline{H_{1} H_{2}}=\{1\}$. Let $N$ be the preimage of $\bar{N}$. Then $N \cap H_{1}=S_{1}, N \cap H_{2}=S_{2}$ and $N H_{1} \cap N H_{2}=N$.

Lemma 3.6. Let $A$ be a subgroup separable group and $H_{1}, H_{2}$ be finitely generated normal subgroups of $A$ and $H_{1} \cap H_{2}=\{1\}$. Then for each $i$ and each $S_{i} \triangleleft_{f} H_{i}$, there exists $f_{H_{i}}\left(S_{i}\right) \subseteq S_{i}$ such that $f_{H_{i}}\left(S_{i}\right)$ is a characteristic subgroup of finite index in $H_{i}$. Furthermore $A$ has the property

(a) for each $S_{1} \triangleleft_{f} H_{1}, S_{2} \triangleleft_{f} H_{2}$, there exists $N \triangleleft_{f} A$ such that $N \cap H_{1}=$ $f_{H_{1}}\left(S_{1}\right), N \cap H_{2}=f_{H_{2}}\left(S_{2}\right)$ and $N H_{1} \cap N H_{2}=N$.

Proof. Follows from Lemma 3.1 and 3.5.

Lemma 3.7. Let $G=A_{H}^{*} B$ and $M, K$ be subgroups of $A, B$ respectively with $M \cap H=\{1\}=K \cap H$. Let $X=H, M$ or $K$. Suppose for each $S_{X} \triangleleft_{f} X$ there exists $f_{X}\left(S_{X}\right) \subseteq X$ such that $f_{X}\left(S_{X}\right)$ is a characteristic subgroup of $X$. Further suppose that $A$ and $B$ have the following properties:

(a) for each $S_{H} \triangleleft_{f} H, S_{M} \triangleleft_{f} M$, there exists $N_{A} \triangleleft_{f} A$ such that $N_{A} \cap H=$ $f_{H}\left(S_{H}\right), N_{A} \cap M=f_{M}\left(S_{M}\right)$ and $N_{A} M \cap N_{A} H=N_{A}$,

(b) for each $S_{H} \triangleleft_{f} H, S_{K} \triangleleft_{f} K$, there exists $N_{B} \triangleleft_{f} B$ such that $N_{B} \cap H=$ $f_{H}\left(S_{H}\right), N_{B} \cap K=f_{K}\left(S_{K}\right)$ and $N_{B} K \cap N_{B} H=N_{B}$.

Then for each $S_{M} \triangleleft_{f} M$ and $S_{K} \triangleleft_{f} K$, there exists $N \triangleleft_{f} G$ such that $N \cap M=$ $f_{M}\left(S_{M}\right), N \cap K=f_{K}\left(S_{K}\right)$ and $N M \cap N K=N$.

Proof. Let $S_{M} \triangleleft_{f} M, S_{K} \triangleleft_{f} K$ be given. Next we let $S_{H}=H$. By assumption, there exist $N_{A} \triangleleft_{f} A, N_{B} \triangleleft_{f} B$ such that $N_{A} \cap H=f_{H}\left(S_{H}\right), N_{A} \cap M=f_{M}\left(S_{M}\right)$, $N_{A} M \cap N_{A} H=N_{A}$ and $N_{B} \cap H=f_{H}\left(S_{H}\right), N_{B} \cap K=f_{K}\left(S_{K}\right), N_{B} K \cap N_{B} H=$ $N_{B}$. Let $\bar{G}=A / N_{A} \frac{*}{H} B / N_{B}$ where $\bar{H}=H N_{A} / N_{A}=H N_{B} / N_{B}$. Since $\bar{G}$ is residually finite by Theorem 2.2 and $\overline{M K}$ is a finite set, there exists $\bar{N} \triangleleft_{f} \bar{G}$ such that $\bar{N} \cap \overline{M K}=\{1\}$. Let $N$ be the preimage of $\bar{N}$. Then $N \cap M=$ $f_{M}\left(S_{M}\right), N \cap K=f_{K}\left(S_{K}\right)$ and $N M \cap N K=N$.

Lemma 3.8. Let $\left\{A_{i}\right\}, i=1,2, \ldots, n$, be groups and $H_{i-1}, H_{i}$ be subgroups of $A_{i}$ with $H_{i-1} \cap H_{i}=\{1\}$. Suppose for each $i$ and each $S_{i} \triangleleft_{f} H_{i}$, there exists $f_{H_{i}}\left(S_{i}\right) \subseteq S_{i}$ such that $f_{H_{i}}\left(S_{i}\right)$ is a characteristic subgroup of $H_{i}$. Further suppose that each $A_{i}$ has the property

(a) for each $S_{i-1} \triangleleft_{f} H_{i-1}, S_{i} \triangleleft_{f} H_{i}$, there exists $N_{A_{i}} \triangleleft_{f} A_{i}$ such that $N_{A_{i}} \cap$ $H_{i-1}=f_{H_{i-1}}\left(S_{i-1}\right), N_{A_{i}} \cap H_{i}=f_{H_{i}}\left(S_{i}\right)$ and $N_{A_{i}} H_{i-1} \cap N_{A_{i}} H_{i}=N_{A_{i}}$.

Let $E_{n}=A_{1} \underset{H_{1}}{\stackrel{*}{*} A_{2}} \stackrel{*}{{ }_{H_{2}}} \cdots{ }_{H_{n-1}}^{*} A_{n}$. Then $E_{n}$ has the property 
(a') for each $S_{0} \triangleleft_{f} H_{0}, S_{n} \triangleleft_{f} H_{n}$, there exists $N \triangleleft_{f} E_{n}$ such that $N \cap H_{0}=$ $f_{H_{0}}\left(S_{0}\right), N \cap H_{n}=f_{H_{n}}\left(S_{n}\right)$ and $N H_{0} \cap N H_{n}=N$

Proof. We prove by induction on $n$. The case $n=2$ follows from Lemma 37 . Let $n \geq 3$. Then $E_{n}=E_{n-1}{ }_{H_{n-1}}^{*} A_{n}$ where $E_{n-1}=A_{1}{ }_{H_{1}}^{*} A_{2}{ }_{H_{2}}^{*}{ }^{*}{ }_{H_{n-2}}^{*} A_{n-1}$. By the induction hypothesis, for each $S_{0} \triangleleft_{f} H_{0}, S_{n-1} \triangleleft_{f} H_{n-1}$, there exists $N_{E_{n-1}} \triangleleft_{f} E_{n-1}$ such that $N_{E_{n-1}} \cap H_{0}=f_{H_{0}}\left(S_{0}\right), N_{E_{n-1}} \cap H_{n-1}=f_{H_{n-1}}\left(S_{n-1}\right)$ and $N_{E_{n-1}} H_{0} \cap N_{E_{n-1}} H_{n-1}=N_{E_{n-1}}$. By (a), for each $S_{n-1} \triangleleft_{f} H_{n-1}, S_{n} \triangleleft_{f} H_{n}$, there exists $N_{A_{n}} \triangleleft_{f} A_{n}$ such that $N_{A_{n}} \cap H_{n-1}=f_{H_{n-1}}\left(S_{n-1}\right), N_{A_{n}} \cap H_{n}=$ $f_{H_{n}}\left(S_{n}\right)$ and $N_{A_{n}} H_{n-1} \cap N_{A_{n}} H_{n}=N_{A_{n}}$. The result now follows from Lemma 3.7 .

Lemma 3.9. (Kim [7]) Let $G=A_{H}^{*} B$ where $A, B$ are $H$-separable. Suppose for each $N_{H} \triangleleft_{f} H$, there exist $N_{A} \triangleleft_{f} A, N_{B} \triangleleft_{f} B$ such that $N_{A} \cap H=N_{B} \cap H \subseteq$ $N_{H}$. Let $S$ be any subgroup of $B$. If $B$ is $S$-separable, then $G$ is $S$-separable.

Lemma 3.10. Let $\left\{A_{i}\right\}, i=1,2, \ldots, n$, be groups and $H_{i-1}, H_{i}$ be subgroups of $A_{i}$ with $H_{i-1} \cap H_{i}=\{1\}$. Suppose each $A_{i}$ and $H_{i}$ satisfy the hypothesis of Lemma 3.8. Further suppose each $A_{i}$ is $H_{i-1}$-separable and $H_{i}$-separable. Let $E_{n}=A_{1}{ }_{H_{1}}^{*} \cdots{ }_{H_{n-1}}^{*} A_{n}$. Then $E_{n}$ is $H_{0}$-separable and $H_{n}$-separable.

Proof. We prove by induction on $n$. The case $n=2$ follows from Lemma 3.9. Let $n \geq 3$. Then $E_{n}=E_{n-1}{ }_{H_{n-1}}^{*} A_{n}$ as in Lemma 3.8. By induction $E_{n-1}$ is $H_{0}$-separable and $H_{n-1}$-separable. By assumption, $A_{n}$ is $H_{n-1}$-separable and $H_{n}$-separable. By Lemma 3.8, for each $S_{0} \triangleleft_{f} H_{0}, S_{n-1} \triangleleft_{f} H_{n-1}$, there exists $N_{E_{n-1}} \triangleleft_{f} E_{n-1}$ such that $N_{E_{n-1}} \cap H_{0}=f_{H_{0}}\left(S_{0}\right), N_{E_{n-1}} \cap H_{n-1}=f_{H_{n-1}}\left(S_{n-1}\right)$ and $N_{E_{n-1}} H_{0} \cap N_{E_{n-1}} H_{n-1}=N_{E_{n-1}}$. By assumption, for each $S_{n-1} \triangleleft_{f} H_{n-1}$, there exists $N_{A_{n}} \triangleleft_{f} A_{n}$ such that $N_{A_{n}} \cap H_{n-1}=f_{H_{n-1}}\left(S_{n-1}\right)$. Hence by Lemma $3.9, E_{n}$ is $H_{0}$-separable and $H_{n}$-separable.

Theorem 3.11. Let $\left\{A_{i}\right\}, i=1,2, \ldots, n$, be subgroup separable groups and $H_{i-1}, H_{i}$ be finitely generated normal subgroups of $A_{i}$ with $H_{i-1} \cap H_{i}=\{1\}$. Let $E_{n}=A_{1}{ }_{H_{1}}^{*} \cdots{ }_{H_{n-1}}^{*} A_{n}$. Then $E_{n}$ is $\pi_{c}$.

Proof. We use induction on $n$. The case $n=2$ follows from Theorem 3.4. Suppose $n \geq 3$. Then $E_{n}=E_{n-1}{ }_{H_{n-1}}^{*} A_{n}$ as in Lemma 3.8. By induction, $E_{n-1}$ is $\pi_{c}$. By assumption, $A_{n}$ is subgroup separable and hence $\pi_{c}$. By Lemma 3.10, $E_{n-1}$ is $H_{0}$-separable and $H_{n-1}$-separable. Since $A_{n}$ is subgroup separable, then $A_{n}$ is $H_{n-1}$-separable. By Lemma 3.8, for each $S_{0} \triangleleft_{f} H_{0}, S_{n-1} \triangleleft_{f} H_{n-1}$, there exists $N_{E_{n-1}} \triangleleft_{f} E_{n-1}$ such that $N_{E_{n-1}} \cap H_{0}=f_{H_{0}}\left(S_{0}\right), N_{E_{n-1}} \cap H_{n-1}=$ $f_{H_{n-1}}\left(S_{n-1}\right)$ and $N_{E_{n-1}} H_{0} \cap N_{E_{n-1}} H_{n-1}=N_{E_{n-1}}$. By Lemma 3.3, for each $S_{n-1} \triangleleft_{f} H_{n-1}$, there exists $N_{A_{n}} \triangleleft_{f} A_{n}$ such that $N_{A_{n}} \cap H_{n-1}=f_{H_{n-1}}\left(S_{n-1}\right)$. Hence by Theorem 2.3, $E_{n}$ is $\pi_{c}$. 


\section{Polygonal products amalgamating normal subgroups}

In this section we extend Theorem 3.11 to polygonal products of finitely many subgroup separable groups amalgamating normal subgroups with trivial intersections. As a consequence polygonal products of polycyclic-by-finite groups and free-by-finite groups are $\pi_{c}$. A polygonal product can be described as follows: (Kim [7]) Let $P$ be a polygon. To each vertex $v$ of $P$ assign a vertex group $G_{v}$ and to each edge $e$ of $P$ assign an edge group $G_{e}$ together with monomorphisms $\alpha_{e}$ and $\beta_{e}$ embedding $G_{e}$ into the two vertex groups at the end of $e$. The polygonal product $G$ is defined to be the group generated by all the generators of the vertex groups with defining relations given by the defining relations of all the vertex groups together with the relations $g_{e} \alpha_{e}=g_{e} \beta_{e}$ for each $g_{e}$ of $G_{e}$. By abuse of language, we say that $G$ is the polygonal product of the (vertex) groups $G_{0}, G_{1}, \ldots, G_{n}$ amalgamating the (edge) groups $H_{0}, H_{1}, \ldots, H_{n}$ with trivial intersections if $G_{i} \cap G_{i+1}=H_{i}$ and $H_{i-1} \cap H_{i}=1$ where $0 \leq i \leq n$ and $H_{-1}=H_{n+1}$.

Lemma 4.1. Let $G=A_{H}^{*} B$ and $M, K$ be subgroups of $A, B$ respectively with $M \cap H=\{1\}=K \cap H$. Suppose for each $N_{H} \triangleleft_{f} H$ there exist $N_{A} \triangleleft_{f} A, N_{B} \triangleleft_{f} B$ such that $N_{A} \cap H=N_{B} \cap H \subseteq N_{H}$ and $N_{A} H \cap N_{A} M=N_{A}, N_{B} H \cap N_{B} K=N_{B}$. If $A$ is $H, M, M H$-separable and $B$ is $H, K, H K$-separable then $G$ is $M K$ separable.

Proof. Let $g \in G-M K$.

Case 1. $g \in B-K, g \notin H$. Since $B$ is $H, K$-separable, there exists $M_{B} \triangleleft_{f} B$ such that $g \notin M_{B} K, g \notin M_{B} H$. Let $N_{H}=M_{B} \cap H$. By assumption there exist $N_{A} \triangleleft_{f} A, N_{B} \triangleleft_{f} B$ such that $N_{A} \cap H=N_{B} \cap H \subseteq N_{H}$ and $N_{A} H \cap N_{A} M=N_{A}$. Now let $R_{A}=N_{A}, R_{B}=N_{B} \cap M_{B}$. Then $R_{A} \cap H=R_{B} \cap H$. Let $\bar{G}=$ $A / R_{A} \frac{*}{H} B / R_{B}$ where $\bar{H}=H R_{A} / R_{A}=H R_{B} / R_{B}$. Clearly $\bar{G}$ is a homorphic image of $G$ and $\bar{H} \cap \bar{M}=1$. Then $\bar{g} \notin \overline{M K}$. Since $\bar{G}$ is residually finite by Theorem 2.2 and $\overline{M K}$ is finite, there exists $\bar{N} \triangleleft_{f} \bar{G}$ such that $\bar{g} \notin \overline{N M K}$. Let $N$ be the preimage of $\bar{N}$. Then $g \notin N M K$.

Case 2. $g \in A-M, g \notin H$. The proof for this case is similar to the proof of Case 1.

Case 3. $g \in H$. Since $g \notin M K$, we have $g \notin M, g \notin K$. Since $A$ is $M$-separable, $B$ is $K$-separable, there exist $M_{A} \triangleleft_{f} A, M_{B} \triangleleft_{f} B$ such that $g \notin M_{A} M, g \notin M_{B} K$. Let $N_{H}=M_{A} \cap M_{B}$. By assumption, there exists $N_{A} \triangleleft_{f} A, N_{B} \triangleleft_{f} B$ such that $N_{A} \cap H=N_{B} \cap H \subseteq N_{H}$ and $N_{A} H \cap N_{A} M=N_{A}$. Now let $R_{A}=N_{A} \cap M_{A}$ and $R_{B}=N_{B} \cap M_{B}$. Then $R_{A} \cap H=R_{B} \cap H$. Let $\bar{G}=A / R_{A} \frac{*}{H} B / R_{B}$ where $\bar{H}=H R_{A} / R_{A}=H R_{B} / R_{B}$. Then $\bar{H} \cap \bar{M}=1$. Clearly $\overline{M K}$ is finite and $\bar{g} \notin \overline{M K}$. We can now proceed as in Case 1 .

Case 4. $g \notin A \cup B$.

Subcase 1. $\|g\| \geq 3$ or $\|g\|=2$ and $g=b_{1} a_{1}$. We will only consider the case $g=b_{1} a_{1} b_{2} a_{2} \cdots b_{k} a_{k}$ where $a_{i} \in A-H, b_{i} \in B-H, i=1,2, \ldots, k$. The other cases are similar. Since $A$ is $H$-separable, $B$ is $H$-separable, there exist 
$M_{A} \triangleleft_{f} A, M_{B} \triangleleft_{f} B$ such that $a_{i} \notin M_{A} H, b_{i} \notin M_{B} H, i=1,2, \ldots, k$. Let $N_{H}=M_{A} \cap M_{B}$. By assumption, there exist $N_{A} \triangleleft_{f} A, N_{B} \triangleleft_{f} B$ such that $N_{A} \cap H=N_{B} \cap H \subseteq N_{H}$. Now let $R_{A}=N_{A} \cap M_{A}, R_{B}=N_{B} \cap M_{B}$. Then $R_{A} \cap H=R_{B} \cap H$. Let $\bar{G}=A / R_{A}{ }_{H}^{*} B / R_{B}$ where $\bar{H}=H R_{A} / R_{A}=H R_{B} / R_{B}$. Clearly $\|\bar{g}\|=\|g\|$ and hence $\bar{g} \notin \overline{M K}$. Furthermore $\overline{M K}$ is finite. We can now proceed as in Case 1.

Subcase 2. $\|g\|=2$ and $g=a b$ where $a \in A-H, b \in B-H$. Now $g \notin M K$ if and only if $a \notin M H$ or $a=m h$ and $h b \notin K$ where $m \in M$, $h \in H$. Suppose $a \notin M H$. Since $A$ is $M H$-separable, $B$ is $H$-separable, there exist $M_{A} \triangleleft_{f} A, M_{B} \triangleleft_{f} B$ such that $a \notin M_{A} M H$ and $b \notin M_{B} H$. Let $N_{H}=M_{A} \cap M_{B}$. By assumption, there exist $N_{A} \triangleleft_{f} A, N_{B} \triangleleft_{f} B$ such that $N_{A} \cap H=N_{B} \cap H \subseteq N_{H}$. Let $R_{A}=N_{A} \cap M_{A}, R_{B}=N_{B} \cap M_{B}$. Then $R_{A} \cap H=R_{B} \cap H$. Let $\bar{G}=A / R_{A} \frac{*}{H} B / R_{B}$ where $\bar{H}=H R_{A} / R_{A}=H R_{B} / R_{B}$. Now $\bar{a} \notin \overline{M H}$ implies $\bar{g} \notin \overline{M K}$. Furthermore $\overline{M K}$ is finite. We can now proceed as in Case 1.

Suppose $a=m h$ and $h b \notin K$. Since $A$ is $H$-separable, $B$ is $H, K$-separable, there exist $M_{A} \triangleleft_{f} A, M_{B} \triangleleft_{f} B$ such that $a \notin M_{A} H, b \notin M_{B} H$ and $h b \notin M_{B} K$. Let $N_{H}=M_{A} \cap M_{B}$. By assumption, there exist $N_{A} \triangleleft_{f} A, N_{B} \triangleleft_{f} B$ such that $N_{A} \cap H=N_{B} \cap H \subseteq N_{H}$ and $N_{A} H \cap N_{A} M=N_{A}, N_{B} H \cap N_{B} K=$ $N_{B}$. Let $R_{A}=N_{A} \cap M_{A}, R_{B}=N_{B} \cap M_{B}$. Then $R_{A} \cap H=R_{B} \cap H$ and $R_{A} H \cap R_{A} M=R_{A}, R_{B} H \cap R_{B} K=R_{B}$. Let $\bar{G}=A / R_{A} \frac{*}{H} B / R_{B}$ where $\bar{H}=H R_{A} / R_{A}=H R_{B} / R_{B}$. Now $\bar{g} \notin \overline{M K}$, for otherwise, $\bar{a}=\bar{m}_{1} \bar{h}_{1}$ and $\bar{h}_{1} \bar{b} \in \bar{K}$ where $\bar{m}_{1} \in \bar{M}, \bar{h}_{1} \in \bar{H}$. Since $\bar{M} \cap \bar{H}=\{1\}$ and $\bar{a}=\bar{m} \bar{h}$, we have $\bar{m}_{1}=\bar{m}, \bar{h}_{1}=\bar{h}$. But this implies that $\overline{h b} \in \bar{K}$, a contradiction. Furthermore $\overline{M K}$ is finite. We can now proceed as in Case 1 . This completes the proof of the lemma.

Lemma 4.2. Let $G=A_{H}^{*} B$ and $M, K$ be subgroups of $A, B$ respectively with $M \cap H=\{1\}=K \cap H$. Suppose $A, B, M, K$ satisfy the hypothesis of Lemma 4.1. Then $G$ is $M * K$-separable.

Proof. Let $g \in G-(M * K)$.

Case 1. $g \in H$. Since $A$ is $M$-separable, $B$ is $K$-separable, there exist $M_{A} \triangleleft_{f} A, M_{B} \triangleleft_{f} B$ such that $g \notin M_{A} M, g \notin M_{B} K$. Let $N_{H}=M_{A} \cap M_{B}$. By assumption, there exist $N_{A} \triangleleft_{f} A, N_{B} \triangleleft_{f} B$ such that $N_{A} \cap H=N_{B} \cap H \subseteq N_{H}$ and $N_{A} H \cap N_{A} M=N_{A}$ and $N_{B} H \cap N_{B} K=N_{B}$. Let $R_{A}=N_{A} \cap M_{A}$ and $R_{B}=N_{B} \cap M_{B}$. Then $R_{A} \cap H=R_{B} \cap H$. Clearly $g \notin R_{A} M$ and $g \notin R_{B} K$. Let $\bar{G}=A / R_{A} \frac{*}{H} B / R_{B}$ where $\bar{H}=H R_{A} / R_{A}=H R_{B} / R_{B}$. Then $\bar{H} \cap \bar{M}=1$ and $\bar{H} \cap \bar{K}=1$. Hence $\bar{g} \notin \bar{M} * \bar{K}$. Since $\bar{G}$ is subgroup separable by Theorem 2.2 and $\bar{M} * \bar{K}$ is finitely generated, there exists $\bar{N} \triangleleft_{f} \bar{G}$ such that $\bar{g} \notin \bar{N}(\bar{M} * \bar{K})$. Let $N$ be the preimage of $\bar{N}$. Then $g \notin N(M * K)$.

Case 2. $\|g\| \geq 1$. We will only consider the case $g=a_{1} b_{1} a_{2} b_{2} \cdots a_{n} b_{n}$ where $a_{i} \in A-H, b_{i} \in B-H, i=1,2, \ldots, n$. The other cases are similar. Now $g \notin M * K$ if and only if there exist $m_{i}, k_{i}, h_{i}$ and $h_{i}^{\prime}$ where $m_{i} \in M, k_{i} \in K$ 
and $h_{i}, h_{i}^{\prime} \in H$ such that one of the following is true:

(1) $a_{1} \notin M H$

or (1') $a_{1}=m_{1} h_{1}$ but $h_{1} b_{1} \notin K H$

or (2) $a_{1}=m_{1} h_{1}, h_{1} b_{1}=k_{1} h_{1}^{\prime}$ but $h_{1}^{\prime} a_{2} \notin M H$

or $\left(2^{\prime}\right) a_{1}=m_{1} h_{1}, h_{1} b_{1}=k_{1} h_{1}^{\prime}, h_{1}^{\prime} a_{2}=m_{2} h_{2}$ but $h_{2} b_{2} \notin K H$

or $(n) a_{1}=m_{1} h_{1}, h_{1} b_{1}=k_{1} h_{1}^{\prime}, \ldots, h_{n-1} b_{n-1}=k_{n-1} h_{n-1}^{\prime}$ but $h_{n-1}^{\prime} a_{n} \notin M H$

or $\left(n^{\prime}\right) a_{1}=m_{1} h_{1}, h_{1} b_{1}=k_{1} h_{1}^{\prime}, \ldots, h_{n-1} b_{n-1}=k_{n-1} h_{n-1}^{\prime}, h_{n-1}^{\prime} a_{n}=m_{n} h_{n}$

but $h_{n} b_{n} \notin K$

Let $s$ be the smallest integer such that $(s)\left(\right.$ or $\left.\left(s^{\prime}\right)\right)$ is true. Now the $m_{j}, k_{j}, h_{j}, h_{j}^{\prime}$ are uniquely determined because $M \cap H=\{1\}=K \cap H$. Since $A$ is $H, M H$-separable, $B$ is $H$-separable, there exist $M_{A} \triangleleft_{f} A, M_{B} \triangleleft_{f} B$ such that $a_{i} \notin M_{A} H, b_{i} \notin M_{B} H$ and $h_{s-1}^{\prime} a_{s} \notin M_{A} M H$. Let $N_{H}=M_{A} \cap M_{B}$. By assumption, there exist $N_{A} \triangleleft_{f} A, N_{B} \triangleleft_{f} B$ such that $N_{A} \cap H=N_{B} \cap H \subseteq N_{H}$ and $N_{A} H \cap N_{A} M=N_{A}, N_{B} H \cap N_{B} K=N_{B}$. Let $R_{A}=N_{A} \cap M_{A}, R_{B}=N_{B} \cap M_{B}$. Then $R_{A} \cap H=R_{B} \cap H$ and $R_{A} H \cap R_{A} M=R_{A}, R_{B} H \cap R_{B} K=R_{B}$. Let $\bar{G}=A / R_{A} \frac{*}{H} B / R_{B}$ where $\bar{H}=H R_{A} / R_{A}=H R_{B} / R_{B}$. Clearly $\|g\|=\|\bar{g}\|$. Suppose $\bar{g} \in \bar{M} * \bar{K}$. Then there exist $\bar{t}_{i} \in \bar{M}, \bar{u}_{i} \in \bar{K}$ and $\bar{v}_{i}, \bar{v}_{i}^{\prime} \in \bar{H}$ such that $\bar{a}_{1}=\bar{t}_{1} \bar{v}_{1}, \bar{v}_{1} \bar{b}_{1}=\bar{u}_{1} \bar{v}_{1}^{\prime}, \ldots, \bar{v}_{s-1} \bar{a}_{s}=\bar{t}_{s} \bar{v}_{s}, \ldots, \bar{v}_{n-1} \bar{a}_{n}=\bar{t}_{n} \bar{v}_{n}$ and $\bar{v}_{n} \bar{b}_{n}=\bar{u}_{n}$. Since $\bar{M} \cap \bar{H}=\{1\}=\bar{K} \cap \bar{H}$, we have $\bar{t}_{i}=\bar{m}_{i}, \bar{v}_{i}=\bar{h}_{i}, \bar{u}_{i}=\bar{k}_{i}, \bar{v}_{i}^{\prime}=\bar{h}_{i}^{\prime}$ for $i=1,2, \ldots, s-1$. But then $\bar{h}_{s-1}^{\prime} \bar{a}_{s} \in \overline{M H}$ a contradiction. Therefore $\bar{g} \notin \bar{M} * \bar{K}$. Furthermore $\bar{G}$ is subgroup separable by Theorem 2.2 and $\bar{M} * \bar{K}$ is finitely generated. We can now proceed as in Case 1 . This completes the proof of the lemma.

Lemma 4.3. Let $\left\{A_{i}\right\}, i=1,2, \ldots, n$, be groups and $H_{i-1}, H_{i}$ be subgroups of $A_{i}$ with $H_{i-1} \cap H_{i}=\{1\}$. Suppose each $A_{i}$ and $H_{i}$ satisfy the hypothesis of Lemma 3.8. Further suppose each $A_{i}$ is $H_{i-1}$-separable, $H_{i}$-separable, $H_{i-1} H_{i}$ -

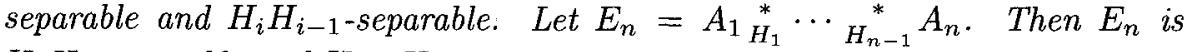
$H_{0} H_{n}$-separable and $H_{0} * H_{n}$-separable.

Proof. We prove by induction on $n$. The case $n=2$ follows from Lemmas 4.1 and 4.2. Let $n \geq 3$. Then $E_{n}=E_{n-1}{ }_{H_{n-1}}^{*} A_{n}$ as in Lemma 3.8. By Lemma $3.10, E_{n-1}$ is $H_{0}$-separable and $H_{n-1 \text {-separable. By induction } E_{n-1}}$ is $H_{0} H_{n-1}$-separable. By assumption, $A_{n}$ is $H_{n-1}$-separable, $H_{n}$-separable and $H_{n-1} H_{n}$-separable. By Lemma 3.8, for each $S_{0} \triangleleft_{f} H_{0}, S_{n-1} \triangleleft_{f} H_{n-1}$, there exists $N_{E_{n-1}} \triangleleft_{f} E_{n-1}$ such that $N_{E_{n-1}} \cap H_{0}=f_{H_{0}}\left(S_{0}\right), N_{E_{n-1}} \cap H_{n-1}=$ $f_{H_{n-1}}\left(S_{n-1}\right)$ and $N_{E_{n-1}} H_{0} \cap N_{E_{n-1}} H_{n-1}=N_{E_{n-1}}$. By assumption, for each $S_{n-1} \triangleleft_{f} H_{n-1}, S_{n} \triangleleft_{f} H_{n}$, there exists $N_{A_{n}} \triangleleft_{f} A_{n}$ such that $N_{A_{n}} \cap H_{n-1}=$ $f_{H_{n-1}}\left(S_{n-1}\right), N_{A_{n}} \cap H_{n}=f_{H_{n}}\left(S_{n}\right)$ and $N_{A_{n}} H_{n-1} \cap N_{A_{n}} H_{n}=N_{A_{n}}$. Hence 
by Lemma $4.1, E_{n}$ is $H_{0} H_{n}$-separable and by Lemma $4.2, E_{n}$ is $H_{0} * H_{n^{-}}$ separable.

Lemma 4.4. Let $G=A_{H}^{*} B$ and $M, K$ be subgroups of $A, B$ respectively with $M \cap H=\{1\}=K \cap H$ and $H, M, K$ be finitely generated. Suppose further that $A$ and $B$ satisfy the hypothesis of Lemma 3.7. Then for each $S \triangleleft_{f}(M * K)$, there exists $N \triangleleft_{f} G$ such that $N \cap(M * K)=f_{M * K}(S)$.

Proof. Let $S \triangleleft_{f}(M * K)$ be given. Since $M * K$ is finitely generated, then by Lemma 3.1, there exists a subgroup $f_{M * K}(S) \subseteq S$ such that $f_{M * K}(S)$ is characteristic and of finite index in $M * K$. Let $S_{M}=f_{M * K}(S) \cap M$ and $S_{K}=f_{M * K}(S) \cap K$. Then $S_{M}$ and $S_{K}$ are characteristic subgroups of $M$ and $K$ respectively. Next we let $S_{H}=H$. Since $H$ is finitely generated, then by Lemma 3.1, we have $f_{H}\left(S_{H}\right)=S_{H}=H$. By assumption, there exists $N_{A} \triangleleft_{f} A$ such that $N_{A} \cap M=f_{M}\left(S_{M}\right)=S_{M}, N_{A} \cap H=f_{H}\left(S_{H}\right)=H$ and $N_{A} M \cap N_{A} H=N_{A}$. Similarly there exists $N_{B} \triangleleft_{f} B$ such that $N_{B} \cap K=$ $f_{K}\left(S_{K}\right)=S_{K}, N_{B} \cap H=f_{H}\left(S_{H}\right)=H$ and $N_{B} H \cap N_{B} K=N_{B}$. Since $N_{A} \cap H=H=N_{B} \cap H$, we can form $\bar{G}=A / N_{A} * B / N_{B}$. Clearly $\bar{G}$ is a homomorphic image of $G$. Let $\psi: G \rightarrow \bar{G}$ be the natural map from $G$ to $\bar{G}$.

We first show that $\operatorname{Ker} \psi \cap(M * K) \subseteq f_{M * K}(S)$. Let $g$ be any element with the smallest length $\|g\|$ such that $g \in \operatorname{Ker} \psi \cap(M * K)$ but $g \notin f_{M * K}(S)$. Without loss of generality let $g=m_{1} k_{1} \cdots m_{n} k_{n}$ where $m_{i} \in M, k_{i} \in K$. Then $\bar{g}=\overline{m_{1} k_{1} \cdots m_{n} k_{n}}=1$. Hence $\bar{m}_{i}=1$ or $\bar{k}_{i}=1$ for some $i$. Without loss of generality let $\bar{m}_{i}=1$. Then $m_{i} \in N_{A}$ since $\bar{M}=M N_{A} / N_{A}$. Hence $m_{i} \in N_{A} \cap M=S_{M} \subseteq f_{M * K}(S)$. Now $g=m_{1} k_{1} \cdots m_{i} k_{i} \cdots m_{n} k_{n}=$ $\left(m_{1} k_{1} \cdots m_{i-1} k_{i-1}\right) m_{i}\left(m_{1} k_{1} \cdots m_{i-1} k_{i-1}\right)^{-1}\left(m_{1} k_{1} \cdots m_{i-1} k_{i-1}\right) k_{i} \cdots m_{n} k_{n}$.

But $\left(m_{1} k_{1} \cdots m_{i-1} k_{i-1}\right) m_{i}\left(m_{1} k_{1} \cdots m_{i-1} k_{i-1}\right)^{-1} \in f_{M * K}(S)$. This implies that $g_{1}=\left(m_{1} k_{1} \cdots m_{i-1} k_{i-1}\right) k_{i} \cdots m_{n} k_{n} \notin f_{M * K}(S)$. But $\overline{g_{1}}=\bar{g}=1$ since $\bar{m}_{i}=1$. Therefore $g_{1} \in \operatorname{Ker} \psi \cap(M * K)$. But $\left\|g_{1}\right\|<\|g\|$, which is a contradiction. Thus Ker $\psi \cap(M * K) \subseteq f_{M * K}(S)$.

Next we show $\overline{f_{M * K}(S)} \cap \bar{M}=1$ and $\overline{f_{M * K}(S)} \cap \bar{K}=1$. Let $\bar{y} \in \overline{f_{M * K}(S)} \cap$ $\bar{M}$. Then $\bar{y}=\bar{s}=\bar{m}$ where $s \in f_{M * K}(S)$ and $m \in M$. Hence $\bar{s} \bar{m}^{-1}=1$ which implies that $s m^{-1} \in \operatorname{Ker} \psi \cap(M * K) \subseteq f_{M * K}(S)$. Therefore $m \in$ $f_{M * K}(S) \cap M \subseteq N_{A}$. This implies that $\bar{m}=1$ and hence $\bar{y}=1$. Hence $\overline{f_{M * K}(S)} \cap \bar{M}=1$. Similarly $\overline{f_{M * K}(S)} \cap \bar{K}=1$.

Now $\bar{G}=A / N_{A} * B / N_{B}=A / N_{A} \frac{*}{M}(\bar{M} * \bar{K}) \frac{*}{K} B / N_{B}$. Since $\overline{f_{M * K}(S)} \cap \bar{M}=$ 1, then $\overline{\bar{M}}=\bar{M} \overline{f_{M * K}(S)} / \overline{f_{M * K}(S)} \simeq \bar{M} / \overline{f_{M * K}(S)} \cap \bar{M} \simeq \bar{M}$. Similarly $\overline{f_{M * K}(S)} \cap \bar{K}=1$ implies that $\overline{\bar{K}}=\bar{K} \overline{f_{M * K}(S)} / \overline{f_{M * K}(S)} \simeq \bar{K} / \overline{f_{M * K}(S)} \cap \bar{K} \simeq$ $\bar{K}$. Thus we can form $\overline{\bar{G}}=A / N_{A} * \frac{*}{\bar{M}}(\bar{M} * \bar{K}) / \overline{f_{M * K}(S)} \frac{*}{\bar{K}} B / N_{B}$. Clearly $\overline{\bar{G}}$ is a homomorphic image of $\bar{G}$ and hence $\overline{\bar{G}}$ is a homomorphic image of $G$.

Since $(\bar{M} * \bar{K}) / \overline{f_{M * K}(S)}$ is finite and $\overline{\bar{G}}$ is residually finite by Theorem 2.2 , then there exists $\overline{\bar{N}} \triangleleft_{f} \overline{\bar{G}}$ such that $\overline{\bar{N}} \cap\left((\bar{M} * \bar{K}) / \overline{f_{M * K}(S)}\right)=\{1\}$. Let $N$. be the preimage of $\overline{\bar{N}}$. Clearly $f_{M * K}(S) \subseteq N \cap(M * K)$. Let $g_{2} \in N \cap(M * K)$. 
Since $\overline{\overline{g_{2}}}=1$, we have $\overline{g_{2}} \in \overline{f_{M * K}(S)}$. Let $\overline{g_{2}}=\bar{t}$ where $t \in f_{M * K}(S)$. Hence $\overline{g_{2}} \bar{t}^{-1}=1$ which implies that $g_{2} t^{-1} \in \operatorname{Ker} \psi \cap(M * K) \subseteq f_{M * K}(S)$. Thus $g_{2} \in f_{M * K}(S)$ and $N \cap(M * K)=f_{M * K}(S)$.

Lemma 4.5. Let $\left\{A_{i}\right\}, i=1,2, \ldots, n$, be groups and $H_{i-1}, H_{i}$ be finitely generated subgroups of $A_{i}$ with $H_{i-1} \cap H_{i}=\{1\}$. Suppose each $A_{i}$ and $H_{i}$ satisfy the hypothesis of Lemma 4.3. Let $E_{n}=A_{1}{ }_{H_{1}}^{*}{ }^{*}{ }_{H_{n-1}}^{*} A_{n}$. Then for each $S \triangleleft_{f}\left(H_{0} * H_{n}\right)$, there exists $N_{E_{n}} \triangleleft_{f} E_{n}$ such that $N_{E_{n}} \cap\left(H_{0} * H_{n}\right)=f_{H_{0} * H_{n}}(S)$.

Proof. We prove by induction on $n$. The case $n=2$ follows from Lemma 4.4. Let $n \geq 3$. Then $E_{n}=E_{n-1}{ }_{H_{n-1}}^{*} A_{n}$ as in Lemma 3.8. Let $S \triangleleft_{f}\left(H_{0} * H_{n}\right)$ be given. Since $H_{0} * H_{n}$ is finitely generated, then by Lemma 3.1, there exists a subgroup $f_{H_{0} * H_{n}}(S) \subseteq S$ such that $f_{H_{0} * H_{n}}(S)$ is characteristic in $H_{0} * H_{n}$. Let $S_{0}=f_{H_{0} * H_{n}}(S) \cap H_{0}$ and $S_{n}=f_{H_{0} * H_{n}}(S) \cap H_{n}$. Then $S_{0}$ and $S_{n}$ are characteristic subgroups of $H_{0}$ and $H_{n}$ respectively. Next we let $S_{n-1}=H_{n-1}$. By Lemma 3.8, there exists $N_{E_{n-1}} \triangleleft_{f} E_{n-1}$ such that $N_{E_{n-1}} \cap H_{0}=f_{H_{0}}\left(S_{0}\right)$, $N_{E_{n-1}} \cap H_{n-1}=f_{H_{n-1}}\left(S_{n-1}\right)$ and $N_{E_{n-1}} H_{0} \cap N_{E_{n-1}} H_{n-1}=N_{E_{n-1}}$. By assumption, there exists $N_{A_{n}} \triangleleft_{f} A_{n}$ such that $N_{A_{n}} \cap H_{n}=f_{H_{n}}\left(S_{n}\right), N_{A_{n}} \cap$ $H_{n-1}=f_{H_{n-1}}\left(S_{n-1}\right)$ and $N_{A_{n}} H_{n-1} \cap N_{A_{n}} H_{n}=N_{A_{n}}$. Hence by Lemma 4.4, there exists $N_{E_{n}} \triangleleft_{f} E_{n}$ such that $N_{E_{n}} \cap\left(H_{0} * H_{n}\right)=f_{H_{0} * H_{n}}(S)$. This completes the proof.

Theorem 4.6. Let $\left\{A_{i}\right\}, i=0,1, \ldots, n$, be subgroup separable groups and $H_{i-1}, H_{i}$ be finitely generated normal subgroups of $A_{i}$ with $H_{i-1} \cap H_{i}=\{1\}$ and $H_{-1}=H_{n}$. Let $G$ be the polygonal product of $A_{1}, A_{2}, \ldots, A_{n}$ amalgamating the subgroups $H_{0}, H_{1}, \ldots, H_{n}$. Then $G$ is $\pi_{c}$.

Proof. Let $E=A_{1}{ }_{H_{1}}^{*} A_{2} \stackrel{*}{H_{2}} \cdots{ }_{H_{n-2}}^{*} A_{n-1}$ and $F=A_{0}{ }_{H_{n}}^{*} A_{n}$. By Theorem 3.11, $E$ and $F$ are $\pi_{c}$. By Lemma 4.3, $E$ and $F$ are $H_{0} * H_{n}$-separable. By Lemma 4.5 , for any $S \triangleleft_{f}\left(H_{0} * H_{n}\right)$, there exist $N_{F} \triangleleft_{f} F, N_{E} \triangleleft_{f} E$ such that $N_{F} \cap\left(H_{0} * H_{n}\right)=N_{E} \cap\left(H_{0} * H_{n}\right) \subseteq S$. The result now follows from Theorem 2.3.

Since polycyclic-by-finite groups and free-by-finite groups are subgroup separable, from Theorem 4.6, we have the following two corollaries.

Corollary 4.7. Let $G$ be a polygonal product of polycyclic-by-finite or freeby-finite groups amalgamating finitely generated normal subgroups with trivial intersections. Then $G$ is $\pi_{c}$.

Corollary 4.8. (Kim [7]) Let $G$ be a polygonal product of finitely generated abelian groups amalgamating subgroups with trivial intersections. Then $G$ is $\pi_{c}$.

\section{References}

[1] R. B. J. T. Allenby and C. Y. Tang, On the residual finiteness of certain polygonal products, Canad. Math. Bull. 32 (1989), no. 1, 11-17. 
[2] G. Baumslag, On the residual finiteness of generalized free products of nilpotent groups, Trans. Amer. Math. Soc. 106 (1963), 193-209.

[3] A. M. Brunner, M. L. Frame, Y. W. Lee, and N. J. Wielenberg, Classifing the torsion-free subgroups of the Picard group, Trans. Amer. Math. Soc. 282 (1984), 205-235.

[4] B. Fine, J. Howie, and G. Rosenberger, One-relator quotients of cyclics, Proc. Amer. Math. Soc. 102 (1988), 249-254.

[5] M. Hall Jr., Coset representationsin free groups, Trans. Amer. Math. Soc. 67 (1949), 421-432.

[6] A. Karrass, A. Pietrowski, and D. Solitar, The subgroups of polygonal products of groups, (Unpublished manucsript).

[7] G. Kim, On polygonal products of finitely generated abelian groups, Bull. Austral. Math. Soc. 45 (1992), 453-462.

[8] A. I. Mal'cev, On homomorphisms onto finite groups, Ivanov. Gos Ped. Inst. Ucen. Zap. 18 (1958), 49-60.

KOK BIN WONG

InSTITUTE OF MATHEMATICAL SCIENCES

UNIVERSITY OF MALAYA

50603 Kuala Lumpur, Malaysia

E-mail address: kbwong@um.edu.my

Peng Choon Wong

Institute of Mathematical Sciences

UNIVERSITY OF MALAYA

50603 Kuala LuMPUR, Malaysia

E-mail address: wongpc@um.edu.my 